\title{
A Case of Mucous Gland Cyst in Uncinate Process
}

\author{
Chunyu W' ${ }^{1}$, Beibei $W^{1}$, Xiaolong $Y^{2}$, Fen $Z^{1}$, \\ Yuanyang $Z^{1}$, Zhiyun $\mathbf{L}^{1}$ and Qingquan $Z^{1,3 *}$ \\ ${ }^{1}$ Department of Otorhinolaryngology, Zhifu Branch of \\ Yantai Yuhuangding Hospital(Yantai Zhifu Hospital), \\ China \\ ${ }^{2}$ Department of Otorhinolaryngology, Yantai Ocean \\ Hospital, China \\ ${ }^{3}$ Department of Otorhinolaryngology, Yantai \\ Yuhuangding Hospital, China \\ *Corresponding author: Zhang Qingquan, \\ Department of Otolaryngology, Zhifu Branch of Yantai \\ Yuhuangding Hospital, China
}

Received: May 14, 2017; Accepted: J une 22, 2017; Published: June 29, 2017

\section{Abstract}

Background: Mucous gland cyst in uncinate process has not been reported home and abroad.

Method: One case of mucous gland cyst was reported. He had a 5-month history of progressive right nasal obstruction. Sinoscopy revealed a light reddish, rough and tough mass which totally occupied his right limen nasi. CT scan showed a homogeneous mass in the right nasal cavity without bony erosion. During the operation, the tumor was broken when pulled by the cutting forceps and milky fluid flowed out from it. The pedicle of the tumor was found in the right uncinate process.

Result: The pathology report proved the diagnosis of mucous gland cyst.

Conclusion: Mucous gland cyst in uncinate process is a rare disease. Mucous gland cyst in uncinate process is easily misdiagnosed, because of the atypical clinical features and imaging findings. Surgical resection is an effective method for treating large mucous gland cyst in uncinate process. Diagnosis depends on pathology.

Keywords: Mucous gland cyst; Uncinate process; Cystic fluid

\section{Introduction}

Mucous gland cyst in nasal sinus is common, but large mucous gland cyst in uncinate process has not been reported home and abroad. We report on a rare case of mucous gland cyst in uncinate process to improve the awareness of mucous gland cyst in uncinate process.

\section{Case Report}

A patient (46Y, male) with a 5-month history of progressive right nasal obstruction was admitted in June 12, 2015. He appeared rhinobyon on right side because of a cold 5 months ago, His nasal congestion gradually increased, until the right nasal cavity was completely blocked. He rarely had a runny nose, but sometimes had a headache on the right side. Sinoscopy revealed a light reddish, rough and tough mass which totally occupied his right limen nasi and could not be contracted by decongestant. Nasal endoscopy could not enter the right nasal cavity after using decongestant. Sinus Computed Tomography (CT) scan showed a homogeneous mass in the right nasal cavity without bony erosion (Figure 1). Diagnosis: benign tumor of the nasal cavity (right). Making sure no taboo, we arranged endoscopic exploratory operation for this patient in June 15,2015 . During the operation, the tumor was broken when pulled by the cutting forceps. The tumor soon shrunk after about 5-6ml milky fluid flowed out from it. With the assistance of elevator the pedicle of the tumor was found in the right uncinate process (Figure 2) and then was removed (Figure 3). The uncinate process stump was properly handled with electrocautery (Figure 4). Gelatin sponge was used to pack the operation cavity. The pathology report proved the diagnosis of mucous gland cyst. The patient was discharged 5 days later. 12 months after operation, the nasal mucosa was recovered well.

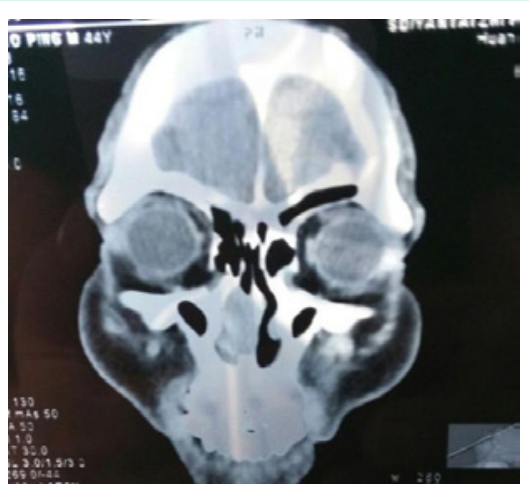

Figure 1: CT showed a soft tissue shadow on the anterior part of the right nasal cavity.

\section{Discussion}

The nasal mucous cyst occurs in the sinus mostly. The most common cause of nasal mucous cystic sinus congestion, it can also occur due to the blockage of opening of serous glands [1]. The cyst in the nasal mucosa is mostly caused by the blockage of the opening of mucous glands or serous glands, Therefore, we believe that in accordance with the naming principle, calling it mucous gland cyst or serous gland cyst is better.

Mucous gland cyst with milky cyst fluid can be found in nasal polyp surgery, and is usually small. In our opinion, the nasal mucosa of multiple nasal polyps is mutually extruded, so that the opening of mucous glands is blocked, leading to the formation of the nasal mucous cyst. The blockage of the opening of mucous glands is seldom happens, without trauma or surgical stimulation. The patient had no history of nasal surgery or nasal trauma. It is extremely rare to have such a huge mucous gland cyst in uncinate process. 


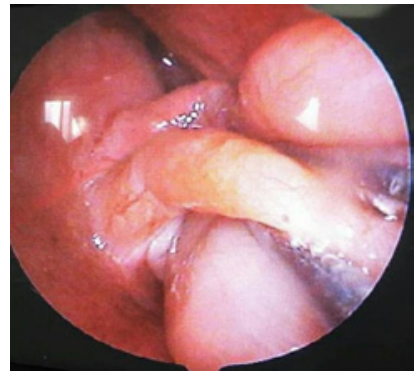

Figure 2: The pedicle of the tumor was found locating in the uncinate process after part of fluid flowed out from the cyst.

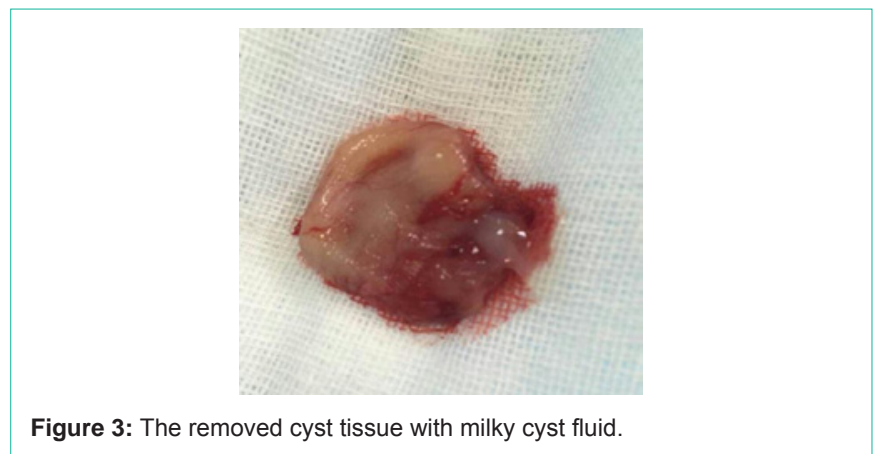

The pedicle of the tumor could not be discovered because the tumor had blocked the anterior naris. In addition, CT showed that the density of the nasal anterior mass was homogeneous, because the anterior part of the mass was ductile. Cyst could not be diagnosed until cyst fluid run from the ruptured mass during operation. Enhanced CT or MRI examination preoperatively is recommended to determine the nature of the tumor [2]. The milky liquid is the sufficient evidence for the diagnosis of mucous gland cyst.

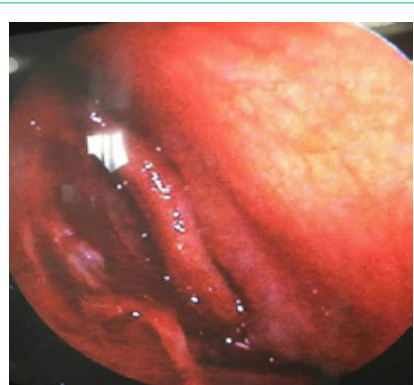

Figure 4: The wound in the uncinate process was visible after excision of the cyst.

\section{Conclusion}

Mucous gland cyst in uncinate process is a rare disease. Mucous gland cyst in uncinate process is easily misdiagnosed, because of the atypical clinical features and imaging findings. Surgical resection is an effective method for treating large mucous gland cyst in uncinate process. Diagnosis depends on pathology.

\section{References}

1. Moriyama H, Nakajima T, Honda Y. Studies on mucocoeles of the ethmoid and sphenoid sinuses:analysis of 47 case [J]. Laryngol Otol.1992; 106: 2327.

2. Malhotrar, Aldp JW, Selvad. Bilateral dynamic proptosis due to frontoethmoidal sinus mucocele [J]. Ophthal Plast Recon str Surg. 2003; 19: 156-157.
Austin J Otolaryngol - Volume 4 Issue 1 - 2017

ISSN : 2473-0645 | www.austinpublishing group.com Qingquan et al. () All rights are reserved
Citation: Chunyu W, Beibei W, Xiaolong Y, Fen Z, Yuanyang Z, Zhiyun L, et al. A Case of Mucous Gland Cyst in Uncinate Process. Austin J Otolaryngol. 2017; 4(1): 1090. 